\title{
Ascariasis hepatobiliar: caso clínico y revisión de la literatura
}

\author{
Argentina Paredes-Soto ${ }^{1}$ y Julio Fernando Toledo-Cuque ${ }^{2 *}$ \\ ${ }^{1}$ Departamento de Medicina Interna; ${ }^{2}$ Departamento de Gastroenterología, Hospital General San Juan de Dios, Ciudad de Guatemala, Guatemala
}

\begin{abstract}
Resumen
Ascaris lumbricoides continúa siendo el principal nematodo que se presenta en humanos. En la mayoría de los casos no se observan síntomas. La presentación hepatobiliar es la entidad clínica menos frecuente, pero se asocia a alta mortalidad. Se documenta el caso de una paciente de 48 años que ingresa con cuadro de dolor abdominal e ictericia. Al evidenciarse en ecografía imagen sugestiva de Ascaris, se realiza colangiopancreatografía retrógrada endoscópica. Se presenta abordaje diagnóstico y terapéutico.
\end{abstract}

Palabras clave: Ascaris lumbricoides. Ascariasis hepatobiliar. Nematodo. Parasitosis biliar.

Hepatobiliary ascariasis: case report and literature review

\begin{abstract}
Ascaris lumbricoides continues to be the main nematode that occurs in humans. In the majority of cases, no symptoms are observed. The hepatobiliary presentation is the less frequent clinical entity, but with high mortality. It is documented the case of a 48-years-old patient who is admitted with abdominal pain and jaundice. Given the ultrasound suggestive image of Ascaris, endoscopic retrograde cholangiopancreatography is performed. The diagnostic and therapeutic approach is presented.
\end{abstract}

Key words: Hepatobiliary ascariasis. Nematode. Biliary parasitosis.

\section{Introducción}

Ascaris lumbricoides es el principal nematodo identificado en la ascariasis en humanos. Esta parasitosis es común en zonas tropicales y malas condiciones de higiene ${ }^{1}$. La mayoría de los casos tiene un curso asintomático y en un bajo número de pacientes los parásitos pueden llegar a invadir los conductos biliares ${ }^{2}$. La ascariasis hepatobiliar es de las manifestaciones menos frecuente, pero que registra una alta mortalidad ${ }^{3}$.

\section{Caso clínico}

Mujer de 48 años sin antecedentes personales patológicos. Acudió al Servicio de Urgencias por cuadro de dolor abdominal de tres días de evolución localizado en cuadrante superior derecho irradiado hacia epigastrio e ictericia.

Paraclínicos: hemograma con hemoglobina $13,23 \mathrm{~g} /$ $\mathrm{dl}$, hematocrito $40.93 \%, 7.39$ leucocitos $/ \mathrm{mm}^{3}$, segmentados $81 \%$, eosinófilos $7.57 \%$, bilirrubina total $0.16 \mathrm{mg} /$

\section{Correspondencia:}

*Julio Fernando Toledo-Cuque

E-mail: dr.juliotoledocuque@gmail.com
Fecha de recepción: 26-03-2019

Fecha de aceptación: 21-04-2019 DOI: 10.24875/END.19000016
Disponible en internet: 06-09-2019

Endoscopia. 2019;31:115-117

www.endoscopia-ameg.com

0188-9893/@ 2019. Asociación Mexicana de Endoscopia Gastrointestinal, publicado por Permanyer México SA de CV, todos los derechos reservados. 


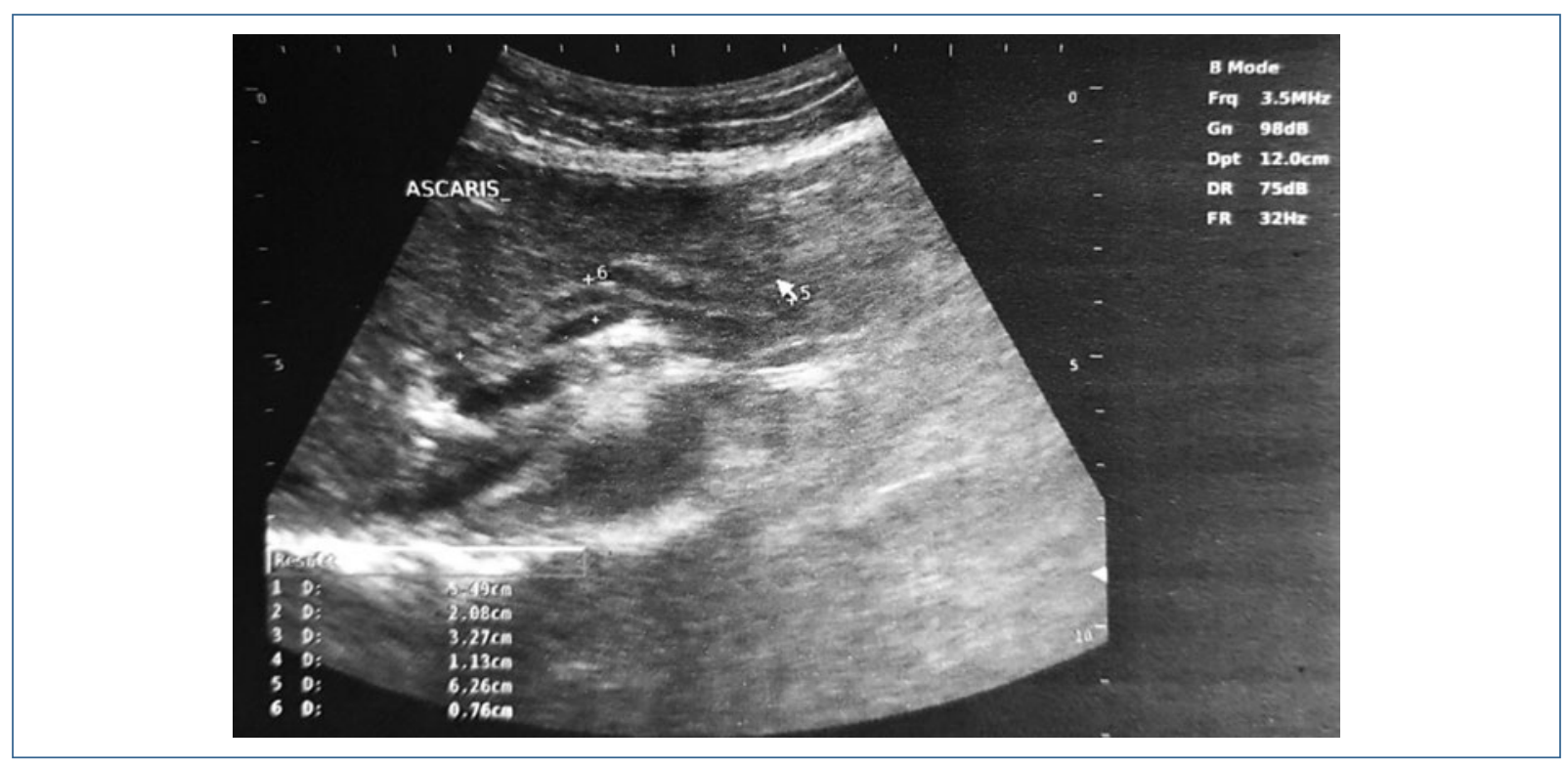

Figura 1. Ecografía de vías biliares.

dl y bilirrubina directa $0.10 \mathrm{mg} / \mathrm{dl}$, gamma glutamil transferasa (GGT) 55U/L, aspartato aminotransferasa (AST) $59 \mathrm{U} / \mathrm{L}$, alanina aminotransferasa (ALT) 52U/L y fosfatasa alcalina (FA) $126 \mathrm{U} / \mathrm{l}$.

Ultrasonido hepático y vías biliares: se evidenció dilatación del colédoco con diámetro máximo de $11.4 \mathrm{~mm}$ y en su interior dos bandas ecogénicas lineales con forma tubular. La vesícula midió 5.4 × $2.0 \times$ $3.2 \mathrm{~cm}$, con pared de $2 \mathrm{~mm}$, sin presencia de lodo biliar o litos (Fig. 1).

Se inició tratamiento con albendazol y posteriormente se realizó colangiopancreatografía retrógrada endoscópica (CPRE). Se observó la vía biliar extrahepática dilatada hasta $20 \mathrm{~mm}$ (Fig. 2), con defecto de llenado de aspecto tubular longitudinal en vía biliar extrahepática. Se extrajo un lito (Fig. 3) y Ascaris de $20 \mathrm{~cm}$ de longitud (Figs. 4 y 5). Su evolución clínica posterior al procedimiento fue favorable.

\section{Discusión}

En áreas endémicas la ascariasis puede llegar a una prevalencia en adultos de hasta el $30 \%$. El ciclo de vida de vida incluye cuatro estadios: embrión, ingestión, migración de la larva y maduración. Los huevos son ingeridos y posteriormente eclosionan, provocando la liberación de larvas que llegan al aparato respiratorio a través de la circulación sanguínea para ser ingeridos nuevamente y habitar el intestino delgado, principalmente el yeyuno ${ }^{4}$. Aunque el mayor número de infecciones son asintomáticas, esto no sucede

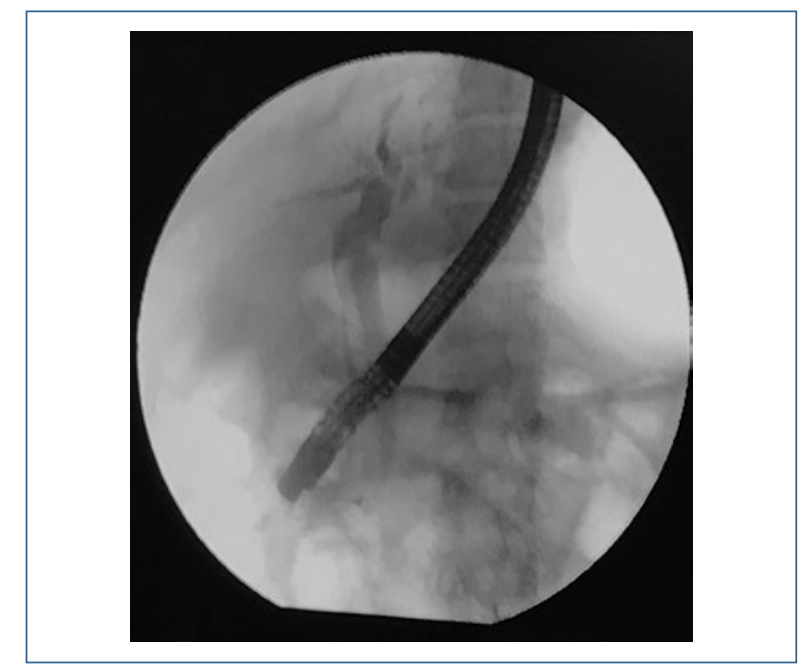

Figura 2. Imagen de fluoroscopia.

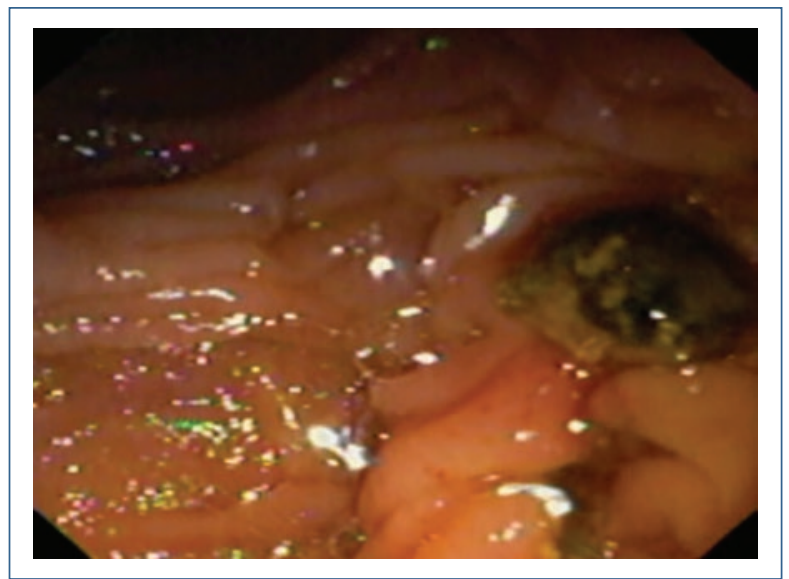

Figura 3. Imagen de lito en endoscopia. 


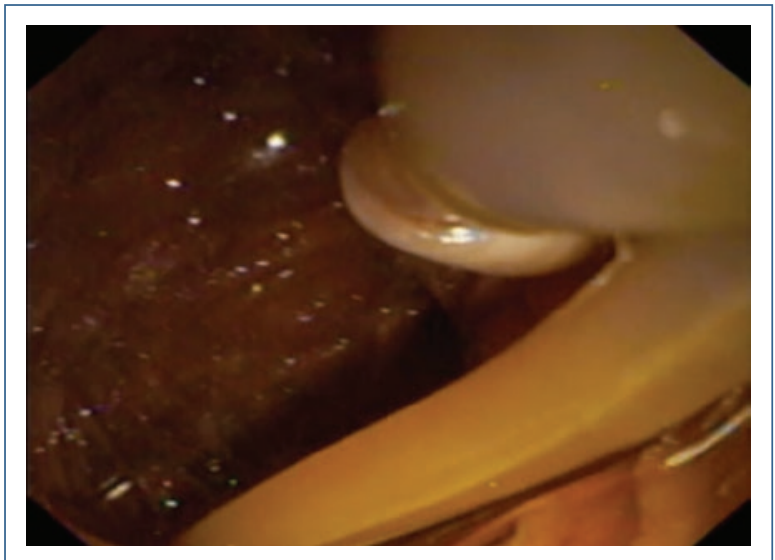

Figura 4. Imagen de extracción de Ascaris lumbricoides.

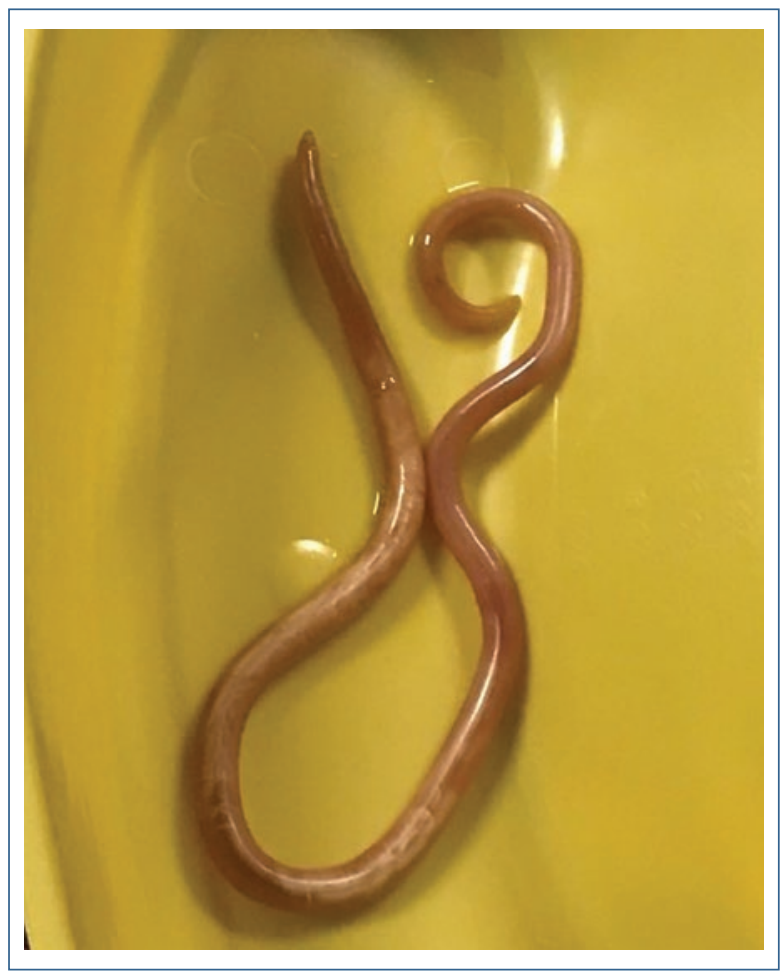

Figura 5. Ascaris lumbricoides.

cuando existe una gran carga de Ascaris lumbricoides. Existen múltiples manifestaciones de la infección y la obstrucción biliar es una complicación importante, ya que el parásito puede migrar a conducto biliar o pancreático y generar colelitiasis, colecistitis aguda, coledocolitiasis, pancreatitis aguda 0 colangitis ascendente ${ }^{5,6}$

La presencia del parásito produce una respuesta inflamatoria intensa que provoca necrosis ductal, calcificaciones, litiasis, estenosis y fibrosis, que causan las distintas manifestaciones. La actividad elevada de la glucuronidasa de los parásitos desconjuga la bilirrubina y ayuda a la formación de litos de pigmento.

El cuadro clínico se caracteriza por dolor abdominal tipo cólico en hipocondrio derecho, vómitos y la evacuación de helmintos por las heces. El tratamiento se enfoca en el síndrome que desarrolle el paciente, su objetivo es la parálisis del helminto para su posterior expulsión. Los fármacos antihelmínticos efectivos son: pamoato de pirantel, mebendazol, albendazol e ivermectina. Los tratamiento invasivos se reservan para cuando ocurre alguna complicación, como colangitis supurativa, o el helminto no se mueva después de tres semanas de seguimiento ${ }^{4}$.

\section{Financiamiento}

La presente investigación no ha recibido ayudas específicas provenientes de agencias del sector públi$\mathrm{co}$, sector comercial o entidades sin ánimo de lucro.

\section{Conflicto de intereses}

Los autores declaran no tener conflicto de intereses.

\section{Bibliografía}

1. Das A. Hepatic and biliary ascariasis. J Glob Infect Dis. 2014;6(2):65.

2. de la Fuente-Lira M, Molotla-Xolalpa C, Rocha-Guevara ER. Ascariasis biliar. Reporte de un caso y revisión en la literatura. Cir Cir. 2006;74(3):195-8.

3. Murillo Castillo E, López González A. Ascariasis hepatobiliar: informe de caso TT. Rev Med Hondur. 2011:79(2):75-8.

4. Khuroo MS, Rather AA, Khuroo NS, Khuroo MS. Hepatobiliary and pancreatic ascariasis. World J Gastroenterol. 2016;22(33):7507-17.

5. Silva AVM da, Massara CL. Ascaris lumbricoides. Parasitol Humana 2005;253-60.

6. Chakrabarti I, De A, Giri A, Roy A. Radio-pathological diagnosis of hepatobiliary ascariasis: A rare entity. J Cytol. 2011;28(3):114. 